\section{A model for computing the dual stiffness matrix of the human knee joint}

\author{
Cristiano Enea, Ettore Pennestrì and Pier Paolo Valentini
}

Proc IMechE Part K:

Julti-body Dynamics

227(4) 407-4I5

(C) IMechE 2013

Reprints and permissions:

sagepub.co.uk/journalsPermissions.nav DOI: 10.1 I77/|4644|93134877|7

pik.sagepub.com

(3)SAGE

\begin{abstract}
This paper discusses an application of dual algebra to the analysis of human knee joint stiffness matrix. According to the proposed methodology, the general stiffness matrix characterizing the elasticity properties of the knee ligaments is reduced to a dual diagonal stiffness matrix. For this purpose, the similarity transform was extended to the field of dual numbers. The theoretical framework proposed seems particularly useful when comparing stiffness matrices obtained from different experimental setup and within different Cartesian coordinate systems.
\end{abstract}

\title{
Keywords
}

Human knee joint stiffness, center of stiffness, biomechanics, dual quaternions, Clifford algebra

Date received: 29 January 2013; accepted: 2 April 2013

\section{Introduction}

From a biomechanical point of view, the knee joint is an elasto-kinematic structure. It means that the relative motion between the bones is influenced not only by the contact shapes but also by the soft tissues contribution. For this reason, the actual screw movement of the joint is dependent from the elastic compliance. In the human knee joint, the main contribution to the stability and to the stiffness properties is due to the presence of the ligaments.

Starting from the physical and mechanical characterization of these structure, ${ }^{1,2}$ each ligament is modeled by means of a line spring or a series of line springs. The tibia and the femur are considered rigid bodies.

In the last 10 years, literature has recorded several investigations on the knee joint elasticity, and an exhaustive review is outside the scope of this paper.

More recent contributions on knee biomechanics are due to Abdel-Nasser et al. ${ }^{3}$ They modeled the ligament structures by means of the absolute nodal coordinate formulation (ANCF). The near incompressibility of the ligaments was considered by introducing a Neo-Hookean strain energy function.

Guess $^{4}$ proposed a multibody dynamics model where the ligaments were represented with nonlinear spring-damper elements with insertions and zero-load lengths derived from experimental measurements. The menisci elasticity was represented by $6 \times 6$ stiffness matrices. The model was validated also using data gathered from a loaded cadaver knee.

In vitro measurements based on cadaver experiments are altered by many factors such as the absence of muscles interaction. For this reason, modern techniques focus on in vivo validation. McFaull and Lamontagne $^{5}$ proposed an experimental procedure to obtain the in vivo passive elastic moment on a knee joint and an equation to estimate the mean angular damping coefficient.

Recently, an in vivo-validated 3D knee dynamic model has been proposed by Akbar et al. ${ }^{6}$ An equivalent stiffness was introduced to simplify a Kelvin viscoelastic model of the cartilage. Ligaments were modeled as bundle of nonlinear tensile springs. The muscles were modeled as strings with a prescribed tension.

Using static radiography and dynamic fluoroscopy, Saevarsson et al. ${ }^{7}$ measured knee kinematics after total knee arthroplasty. Great behavior differences were detected in static and dynamic kinematics of the knee joint.

For a given mechanical system, in the Cartesian Space, the stiffness properties can be represented by a symmetric $6 \times 6$ matrix. First, Ball $^{8}$ and then Dimentberg ${ }^{9}$ used the screw theory for the analysis of rigid body motion and the computation of the stiffness matrix associated to a rigid body suspended by linear springs connected in parallel.

Department of Industrial Engineering, University of Rome "Tor Vergata", Rome, Italy

\section{Corresponding author:}

Ettore Pennestrì, Dipartimento di Ingegneria Industriale, Università di Roma Tor Vergata, Via del Politecnico, I 00133 Roma, Italy. Email: pennestri@mec.uniroma2.it 
In a thoughtful contribution on modeling of elastically suspended bodies, Fasse ${ }^{10}$ represented the strain potential energy by a quadratic function of dual quaternion displacement. Viscous constitutive equations expressed in terms of dual numbers were also introduced.

Because of the arbitrariness of the reference frame adopted in the analysis, the results of different works are not directly comparable. From this consideration, there is the necessity of adopting analytical approaches, based on a normalized form of the stiffness matrix, to obtain frame-independent stiffness properties. ${ }^{10}$

A starting point of these analytical approaches is represented by the concept of center of stiffness proposed by Loncaric ${ }^{11,12}$ to decouple the stiffness associated with the translational and rotational motions.

The Principle of Transference of dual algebra allows an extension of this concept to the dual space. By associating a dual vector to each line spring of the system, a $3 \times 3$ symmetric dual matrix stiffness is obtained. Subsequently, by computing its dual eigenvalues and dual eigenvectors ${ }^{13}$ it is possible to evaluate a normalized form of the stiffness dual matrix which decouples maximally the translational stiffness from the rotational one. ${ }^{14}$

In this investigation, a normalized form of the human knee joint stiffness matrix is presented. The matrix components are expressed within the framework of Clifford dual algebra. The model is linear and valid for small motions about equilibrium configurations.

In particular, within the hypotheses of linear elasticity and small displacements, an attempt is made to analyze the dual stiffness matrix of the human knee joint. Our methodology has been applied to the stiffness models of Crowninshield et al. ${ }^{2}$ and AbdelRahaman and Hefzy. ${ }^{1}$

The authors are aware that a linear elastic model cannot faithfully represent the complex behavior of the knee ligaments. This is also witnessed by the experimental evidence and by the nonlinear functions proposed to represent the elasticity of a knee joint that undergoes large rotations.

However, under small displacements about equilibrium configurations, the constant stiffness approximation is considered feasible for a preliminary analysis.

The computation of the dual stiffness matrix has been implemented using Ch programming language. ${ }^{15,16}$

\section{The stiffness matrix}

A linear elastic system can be modeled as a rigid body connected to the ground by springs arranged in parallel. In such a system, the stiffness matrix $[K]$ is a linear map which relates wrench and twist. Such linear map is defined as follows

$$
\{\mathrm{d} W\}=[K]\{d q\}
$$

where $\{\mathrm{d} W\}_{6 \times 1}$ is the infinitesimal change in load (wrench) while $\{d q\}_{6 \times 1}$ is the infinitesimal displacement (twist). The stiffness matrix $[K]$ is a $6 \times 6$ symmetric matrix that can be partitioned as follows

$$
[K]=\left[\begin{array}{ll}
K_{t} & K_{c} \\
K_{c}^{T} & K_{r}
\end{array}\right]
$$

In this partition, the blocks $\left[K_{t}\right]$ and $\left[K_{r}\right]$ are $3 \times 3$ symmetric matrices; generally $\left[K_{t}\right]$ is defined the translational stiffness matrix while $\left[K_{r}\right]$ is the rotational stiffness matrix. The $3 \times 3$ coupling stiffness matrix $\left[K_{c}\right]$ is a non-symmetric. Each subscripted quantity is origin dependent; besides, considering an elastic system in a loaded configuration, the stiffness matrix results to be asymmetric. ${ }^{17}$

\section{Linear and torsional springs}

Consider the system represented in Figure 1, showing a rigid body suspended by linear springs connected in parallel.

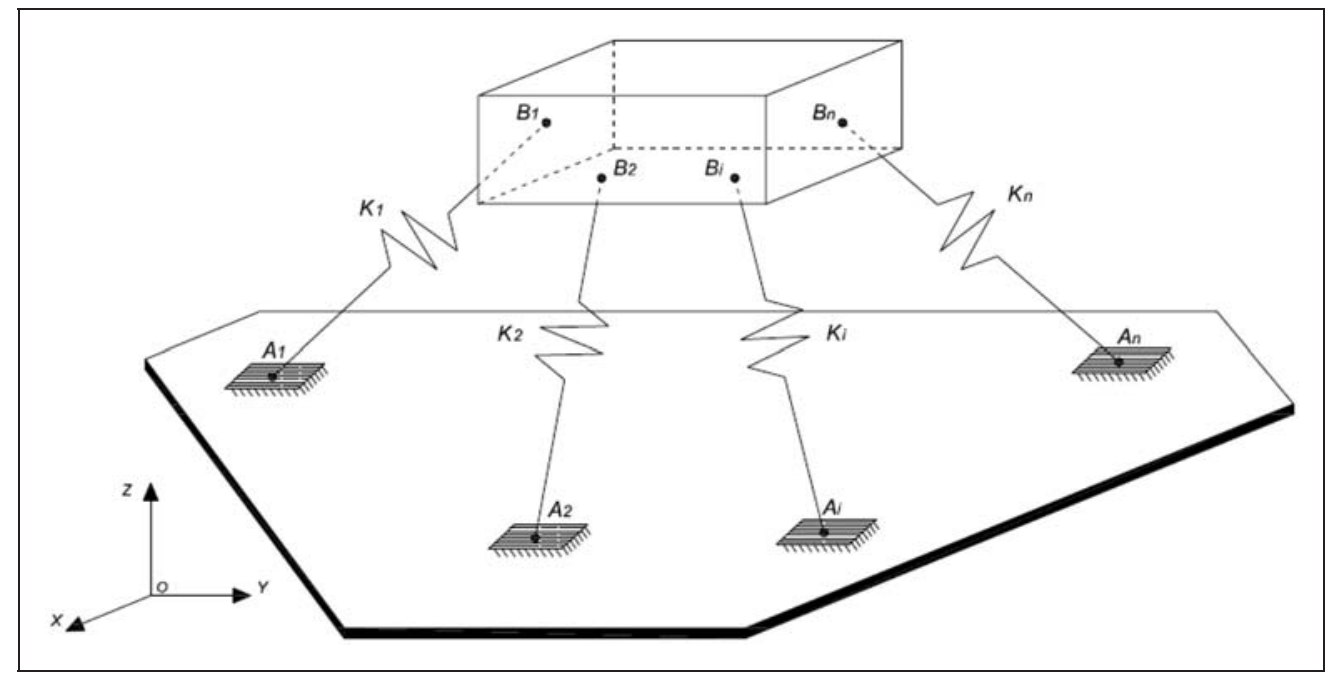

Figure I. Rigid body suspended by line springs. 
When the system is in an unloaded configuration, the springs are not stretched. The stiffness associated with the $i$ th spring is

$$
\left[K_{i}\right]=k_{i}\left\{S_{i}\right\}\left\{S_{i}\right\}^{T}
$$

where $k_{i}$ is the scalar spring stiffness

$$
\left\{S_{i}\right\}=\left\{\begin{array}{c}
\left\{s_{i}\right\} \\
\left\{O A_{i}\right\} \times\left\{s_{i}\right\}
\end{array}\right\}, \quad\left\{s_{i}\right\}^{T}\left\{s_{i}\right\}=1
$$

denotes the unit line vector, with $\left\{s_{i}\right\}$ unit vector, in the direction of the $i$ th spring line of action.

The stiffness matrix of the whole spring system, characterized by $n$ linear springs connected in parallel, can be computed as follows

$$
[K]=\sum_{i=1}^{n}\left[K_{i}\right]=\sum_{i=1}^{n} k_{i}\left\{S_{i}\right\}\left\{S_{i}\right\}^{T}
$$

Similarly, the stiffness matrix for a rigid body attached to torsional springs of stiffness $k_{i}$ $(i=1,2, \ldots, n)$ is expressed by

$$
[K]=\sum_{i=1}^{n} k_{i}\left\{S_{i}\right\}\left\{S_{i}\right\}^{T}=\sum_{i=1}^{n} k_{i}\left[\begin{array}{cc}
{[0]} & {[0]} \\
{[0]} & {\left[\left\{s_{i}\right\}\left\{s_{i}\right\}^{T}\right]}
\end{array}\right]
$$

\section{The center of stiffness}

The elements of the stiffness matrix depend on the choice of coordinate frame. Loncaric ${ }^{11,12}$ has shown that it is possible to distinguish a particular and suitable coordinate frame which led us to define the center of stiffness.

Let us consider a symmetric stiffness matrix $[K]$ expressed by

$$
[K]=\left[\begin{array}{cc}
K_{t} & K_{c} \\
K_{c}^{T} & K_{r}
\end{array}\right]
$$

where the diagonal blocks, $\left[K_{t}\right]$ and $\left[K_{r}\right]$, are $3 \times 3$ symmetric ones while the off-diagonal blocks are non-symmetric. If $\operatorname{tr}\left[K_{t}\right]$ is not an eigenvalue of $\left[K_{t}\right]$, then $\left[K_{c}\right]$, the coupling stiffness matrix is symmetric at an unique point known as center of stiffness. ${ }^{11,12}$ In particular, assuming the origin of the coordinate frame in this point the maximum decoupling between translational and rotational stiffness is achieved.

Let us denote, respectively, by $[R]$ and $\{p\}$ the cosine direction matrix and displacement vector which define the position of a new Cartesian reference frame.

Introduce the $6 \times 6$ adjoint matrix $[\operatorname{adj} H]$

$$
[\operatorname{adj} H]=\left[\begin{array}{cc}
{[R]} & {[\tilde{p}][R]} \\
{[0]} & {[R]}
\end{array}\right]
$$

where $[R]$ is an orthonormal $3 \times 3$ matrix and $[\tilde{p}]$ and the skew-symmetric matrix associated to vector $\{p\}$.

In the new Cartesian reference frame, with the origin in the center of stiffness, the stiffness matrix is

$$
\left[K^{\prime}\right]=[\operatorname{adj} H]^{T}[K][\operatorname{adj} H]
$$

The location of the center of stiffness can be computed from (9) by requiring that the coupling stiffness matrix, $\left[K_{c}\right]$ be symmetric. In particular, assuming the stiffness matrix $[K]$ defined by the following relation

$$
[K]=\left[\begin{array}{llllll}
k_{11} & k_{12} & k_{13} & k_{14} & k_{15} & k_{16} \\
k_{12} & k_{22} & k_{23} & k_{24} & k_{25} & k_{26} \\
k_{13} & k_{23} & k_{33} & k_{34} & k_{35} & k_{36} \\
k_{14} & k_{24} & k_{34} & k_{44} & k_{45} & k_{46} \\
k_{15} & k_{25} & k_{35} & k_{45} & k_{55} & k_{56} \\
k_{16} & k_{26} & k_{36} & k_{46} & k_{56} & k_{66}
\end{array}\right]
$$

through a linear displacement $p$ and an appropriate rotation $R$ of the Cartesian reference frame, we can obtain the following form of the matrix

$$
\left[K^{\prime}\right]=\left[\begin{array}{cccccc}
k_{11}^{\prime} & k_{12}^{\prime} & k_{13}^{\prime} & k_{14}^{\prime} & 0 & 0 \\
k_{12}^{\prime} & k_{22}^{\prime} & k_{23}^{\prime} & 0 & k_{25}^{\prime} & 0 \\
k_{13}^{\prime} & k_{23}^{\prime} & k_{33}^{\prime} & 0 & 0 & k_{36}^{\prime} \\
k_{14}^{\prime} & 0 & 0 & k_{44}^{\prime} & k_{45}^{\prime} & k_{46}^{\prime} \\
0 & k_{25}^{\prime} & 0 & k_{45}^{\prime} & k_{55}^{\prime} & k_{56}^{\prime} \\
0 & 0 & k_{36}^{\prime} & k_{46}^{\prime} & k_{56}^{\prime} & k_{66}^{\prime}
\end{array}\right]
$$

\section{The diagonal form of the stiffness dual matrix}

The Principle of Transference ${ }^{9}$ allows the extension of this algorithm to the dual space. Given a mechanical system composed of a rigid body connected to the ground by springs in parallel, the dual stiffness matrix can be computed. Through an appropriate transform, this stiffness can be expressed in a diagonal form in order to decouple the different stiffness modes. In the following sections, the main steps of the proposed algorithm are presented.

\section{Characterization of the system geometry}

The first step is represented by the characterization of the system geometry such as the insertion points of the springs on the rigid body and to the ground (or on another rigid body assumed to be the fixed element of the system). Once the insertion points have been fixed, a proper dual vector $\left\{\hat{v}_{i}\right\}$ can be associated to each spring of the system. The dual vector is in the form

$$
\left\{\hat{v}_{i}\right\}=\left\{v_{i}\right\}+\varepsilon\left(\left\{O A_{i}\right\} \times\left\{v_{i}\right\}\right)
$$


where the vector $\left\{O A_{i}\right\}$ joins the origin of the coordinate frame to the insertion point of the $i$ th spring to the ground.

\section{Computation of the stiffness dual matrix}

The stiffness $\left[\widehat{K}_{i}\right]$ associated to each spring is expressed by

$$
\left[\hat{K}_{i}\right]=k_{i}\left\{\hat{v}_{i}\right\}\left\{\hat{v}_{i}\right\}^{T}
$$

where $k_{i}$ represents the stiffness of the $i$ th linear spring. Therefore, considering a system composed of two rigid bodies, connected by $n$ linear springs in parallel, the dual stiffness matrix of the entire system can be computed as follows

$$
[\hat{K}]=\sum_{i=1}^{n}\left[\hat{K}_{i}\right]=\sum_{i=1}^{n}\left(k_{i}\left\{\hat{v}_{i}\right\}\left\{\hat{v}_{i}\right\}^{T}\right)
$$

The stiffness matrix is a $6 \times 6$ symmetric one, whereas the dual stiffness matrix is a $3 \times 3$ symmetric expressed by

$$
[\hat{K}]=[K]+\varepsilon\left[K^{o}\right]
$$

where $[K]$ and $\left[K^{\circ}\right]$ are the real and dual parts, respectively.

\section{Computation of the eigenvalues and the eigenvec- tors of the stiffness dual matrix}

After the dual stiffness matrix has been evaluated, its dual eigenvalues $\hat{\lambda}_{i}=\lambda+\varepsilon \lambda^{\circ}$ and the associated dual eigenvectors $\left\{\hat{e}_{i}\right\}=\left\{e_{i}\right\}+\varepsilon\left\{e_{i}^{o}\right\}$ are computed by means of the following equation

$$
[\hat{K}]\left\{\hat{e}_{i}\right\}=\hat{\lambda}\left\{\hat{e}_{i}\right\}
$$

After separating the real and the dual parts, from (16) one obtains

$$
\begin{aligned}
& {[K-\lambda I]\{e\}=\{0\}} \\
& {\left[\begin{array}{cc}
{[K-\lambda I]} & -\{e\} \\
\{e\}^{T} & 0
\end{array}\right]\left\{\begin{array}{l}
e^{o} \\
\lambda^{o}
\end{array}\right\}=\left\{\begin{array}{c}
-\left[K^{o}\right]\{e\} \\
0
\end{array}\right\}}
\end{aligned}
$$

where the normalization condition $\{\hat{e}\}^{T}\{\hat{e}\}=1$ holds.

Once the eigenvalues $\hat{\lambda}$ and the eigenvectors $\{\hat{e}\}$ have been computed, we obtain the orthogonal eigenvector matrix $[\hat{E}]$

$$
[\hat{E}]=\left[\begin{array}{ccc}
\mid & \mid & \mid \\
\left\{\hat{e}_{1}\right\} & \left\{\hat{e}_{2}\right\} & \left\{\hat{e}_{3}\right\} \\
\mid & \mid & \mid
\end{array}\right]
$$

required to diagonalize the dual stiffness matrix $[\hat{K}]$.
By applying the dual similarity transform, one obtains

$$
\left[\hat{K}^{\prime}\right]=[\hat{E}]^{T}[\hat{K}][\hat{E}]
$$

where $\left[\hat{K}^{\prime}\right]$ is a diagonal $3 \times 3$ with the following structure

$$
\left[\widehat{K}^{\prime}\right]=\left[\begin{array}{ccc}
\hat{k}_{1} & 0 & 0 \\
0 & \hat{k}_{2} & 0 \\
0 & 0 & \hat{k}_{3}
\end{array}\right]
$$

\section{Computation of motion parameters from the dual transformation matrix}

The dual matrix $[\hat{E}]$ characterizes the rigid displacement of the Cartesian reference frame into a new position. Within this new Cartesian reference frame, the dual spring stiffness is diagonal. From (18), the dual Euler parameters $\left\{\begin{array}{llll}\hat{e}_{0} & \hat{e}_{1} & \hat{e}_{2} & \hat{e}_{3}\end{array}\right\}$ of this motion are obtained.

Dual angle $\hat{\theta}$ and screw axis, located by the unit dual vector $\hat{h}$, of the finite helical motion can be expressed as functions of dual Euler parameters

$$
\begin{aligned}
& \hat{\theta}=2 \cos ^{-1} \hat{e}_{0} \\
& \hat{h}_{x}=\frac{\hat{e}_{1}}{\sin \frac{\hat{\theta}}{2}}, \quad \hat{h}_{y}=\frac{\hat{e}_{2}}{\sin \frac{\hat{\theta}}{2}}, \quad \hat{h}_{z}=\frac{\hat{e}_{3}}{\sin \frac{\hat{\theta}}{2}}
\end{aligned}
$$

\section{Numerical examples}

The methodology herein discussed has been applied to the knee elastic models of Abdel-Raham and Hefzy ${ }^{1}$ and Crowninshield et al. ${ }^{2}$

The insertion sites are obtained in the Cartesian reference systems shown in Figure 2.

The computation of the dual stiffness matrix, and its following handling, in these examples have been implemented using the Ch programming language, a programming language developed by Cheng, ${ }^{16,18,19}$ making use of the linear dual algebra routines developed by Pennestrì et al. ${ }^{13,20,21}$

\section{Model of Abdel-Raham and Hefzy}

The theory presented in the previous sections has been applied to compute the stiffness dual matrix associated to the knee joint.

A CAD model of the knee joint and its ligaments is shown in Figure 3. In particular, the model proposed by Abdel-Raham and Hefzy ${ }^{1}$ was considered.

The model of knee joint considered is the model proposed by Abdel-Raham and Hefzy. The tibia and the femur are modeled as two rigid bodies; the femur is assumed to be the fixed element while the tibia is the moving one. 


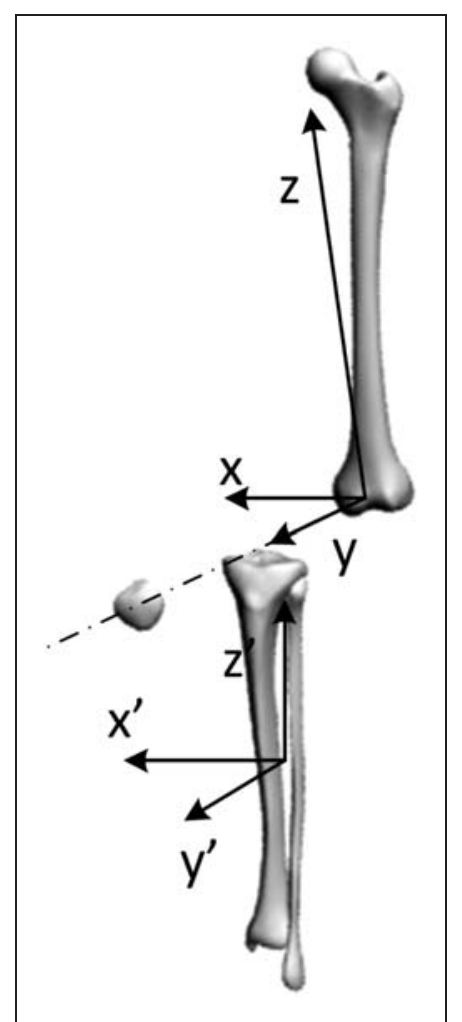

Figure 2. Cartesian reference systems in Abdel-Raham and Hefzy.

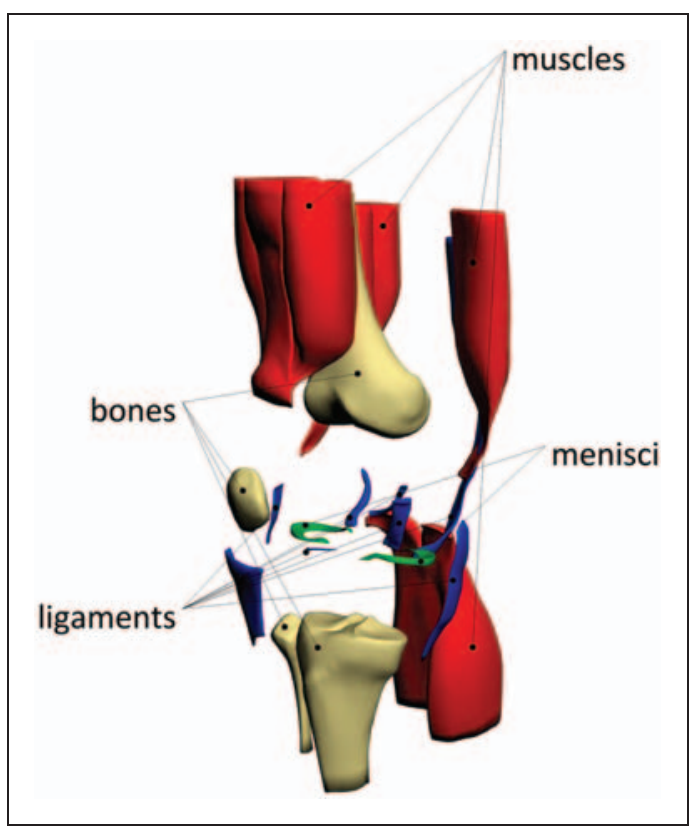

Figure 3. CAD model view of a knee joint.

The cartilage deformation is assumed to be relative small compared with joint motions. Besides, friction forces are neglected because of the low coefficient of friction of the articular surfaces. In such a model, the resistance to motion is essentially due to the ligaments structures. The ligaments are modeled with 12 spring elements, representing the ligaments and the capsular tissue posterior to the knee joint; in particular, the fiber bundles of anterior cruciate ligament (ACL) is represented by two elements and the other two elements represent the posterior cruciate ligament (PCL). The medial collateral ligament (MCL) is modeled as three elements while the lateral collateral ligament (LCL) is represented by one elastic element. Finally, four elements represent the fiber bundles of the posterior part of the capsule (CAP). These elements are represented in Figure 4.

The insertion sites of the ligaments on the femur and the tibia are assumed according with the data available in literature and reported in Table 1.

It is necessary to observe that in this model, the wrapping of the ligaments on the bone structures in not considered. Hence, the ligaments are represented as line elements extending from the femur surface to the tibial one.

Although the force-elongation relationship of the ligaments results to be non-linear globally, we can assume that near the equilibrium position this relationship can be assumed linear. The values of the stiffness coefficients of the spring elements used to model the different ligamentous structures were assumed according to the data available in literature. For completeness, the numerical stiffness data according to Abdel-Rahaman and Hefzy model ${ }^{1}$ have been summarized in Tables 1 and 2, respectively. In the cited bibliographical reference, the ligaments are considered as a neo-Hookean material. Since our analysis is limited to small displacements, it has been assumed that the strain is less than 0.03 . Within this limit, a linear relationship between applied stress and strain is reported by Abdel-Rahaman and Hefzy.

Applying the algorithm presented in the previous section, we are able to compute the stiffness dual matrix $[\hat{K}]$ which is defined as

$$
\begin{aligned}
{[K]=} & {\left[\begin{array}{ccc}
22.788 & -14.239 & -13.968 \\
-14.239 & 38.165 & -20.954 \\
-13.968 & -20.954 & 652.097
\end{array}\right] } \\
& +\varepsilon\left[\begin{array}{ccc}
372.388 & -293.373 & -10700.940 \\
-293.373 & 158.161 & 1460.566 \\
-10700.940 & 1460.566 & -530.549
\end{array}\right]
\end{aligned}
$$

The computation of eigenvalues $\hat{\lambda}$ and eigenvectors $\{\hat{e}\}$ led us to the following results

$$
\begin{aligned}
& \hat{\lambda}_{1}=13.481+\varepsilon(545.937) \\
& \hat{\lambda}_{2}=46.470+\varepsilon(-716.340) \\
& \hat{\lambda}_{3}=653.100+\varepsilon(170.403) \\
& \left\{\hat{e}_{1}\right\}=\left\{\begin{array}{l}
-0.852 \\
-0.522 \\
-0.036
\end{array}\right\}+\left\{\begin{array}{c}
2.339 \\
-2.920 \\
-13.130
\end{array}\right\}
\end{aligned}
$$




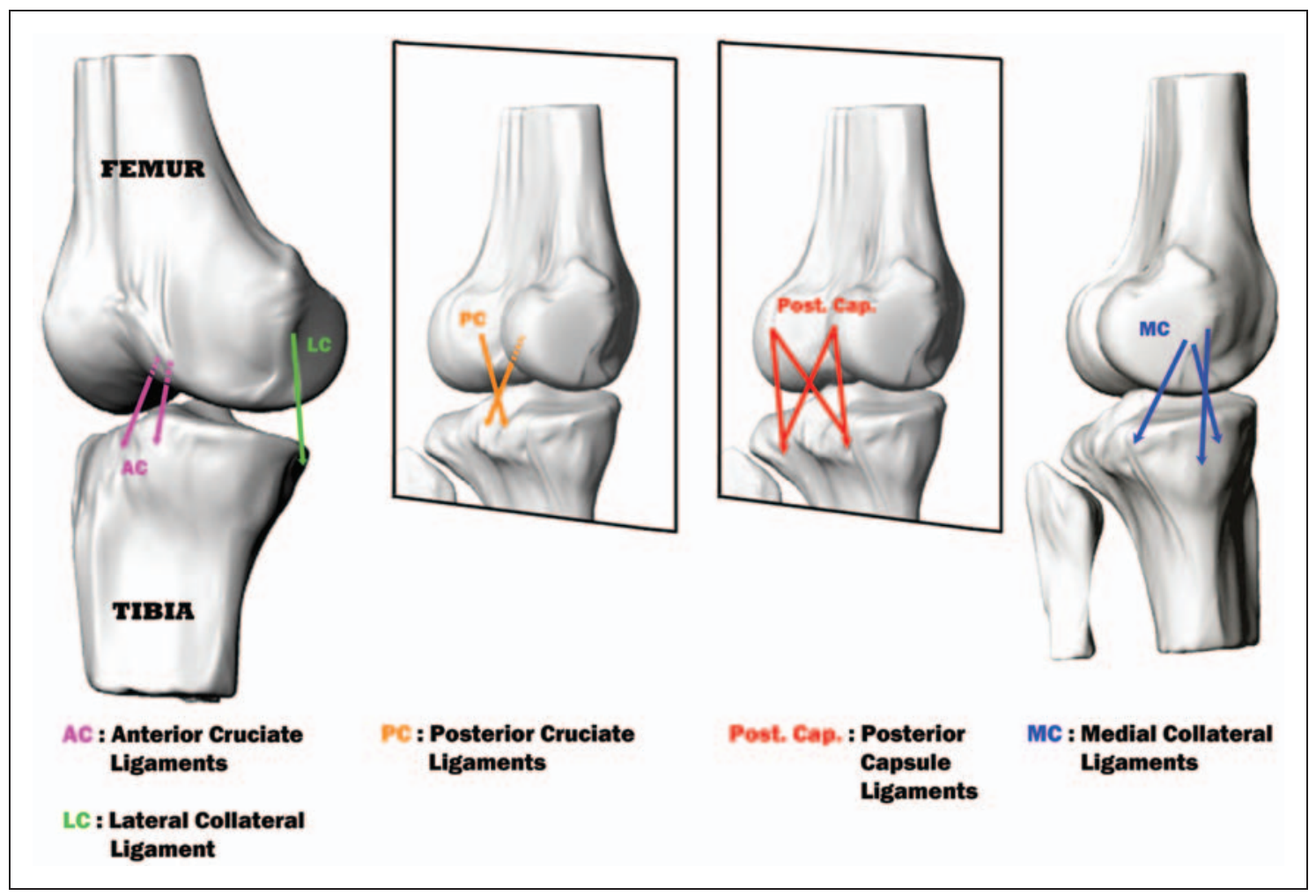

Figure 4. Line vectors associated to the ligamentous structures - model of Abdel-Raham and Hefzy.

Table I. Local attachment coordinates and material properties of the ligamentous structures of the Abdel-Rahaman and Hefzy model.

\begin{tabular}{|c|c|c|c|c|c|c|c|}
\hline & \multicolumn{3}{|c|}{ Femoral attachments } & \multicolumn{3}{|c|}{ Tibial attachments } & \multirow{2}{*}{$\frac{\text { Material propertie }}{K_{\mathrm{I}}\left(\frac{\mathrm{N}}{\mathrm{mm}}\right)}$} \\
\hline & $X_{f}(\mathrm{~mm})$ & $Y_{f}(\mathrm{~mm})$ & $Z_{f}(\mathrm{~mm})$ & $X_{t}(\mathrm{~mm})$ & $Y_{t}(\mathrm{~mm})$ & $Z_{t}(\mathrm{~mm})$ & \\
\hline$A C L$ anterior fibers & 7.25 & -15.6 & 21.25 & -7.0 & 5.0 & 211.25 & 22.5 \\
\hline$A C L$ posterior fibers & 7.25 & -20.3 & 19.55 & 2.0 & 2.0 & 212.25 & 26.3 \\
\hline PCL anterior fibers & -4.75 & -11.2 & 14.05 & 5.0 & -30.0 & 206.25 & 31.3 \\
\hline PCL posterior fibers & -4.75 & -23.2 & 15.65 & -5.0 & -30.0 & 206.25 & 19.3 \\
\hline$M C L$ anterior fibers & -34.75 & -1.0 & 26.25 & -20.0 & 4.0 & 171.25 & 10.0 \\
\hline MCL posterior fibers & -34.75 & -8.0 & 24.25 & -35.0 & -30.0 & 199.25 & 5.0 \\
\hline MCL deep fibers & -34.75 & -5.0 & 21.25 & -35.0 & 0.0 & 199.25 & 5.0 \\
\hline LCL & 35.25 & -15.0 & 21.25 & 45.0 & -25.0 & 176.25 & 10.0 \\
\hline Posterior capsule, medial & -24.75 & -38.0 & 6.25 & -25.0 & -25.0 & 181.25 & 12.0 \\
\hline Posterior capsule, lateral & 25.25 & -35.5 & 8.25 & 25.0 & -25.0 & 181.25 & 12.0 \\
\hline Posterior capsule, obl. poplit. ligament & 25.25 & -35.5 & 8.25 & -25.0 & -25.0 & 181.25 & 3.0 \\
\hline Posterior capsule, arcuate. poplit. ligament & -24.75 & -38.0 & 6.25 & 25.0 & -25.0 & 181.25 & 3.0 \\
\hline
\end{tabular}

The points of attachment coordinates of the ligaments in the Crowninshield et al. model ${ }^{2}$ (see Figure 5) are reported in the Table 2. The ligament stiffness are those reported by Hefzy and Abdel-Rahaman.

$$
\begin{array}{r}
\left\{\hat{e}_{2}\right\}=\left\{\begin{array}{c}
-0.522 \\
0.852 \\
0.017
\end{array}\right\}+\left\{\begin{array}{c}
-3.117 \\
-1.679 \\
-11.386
\end{array}\right\} \\
\left\{\hat{e}_{3}\right\}=\left\{\begin{array}{c}
-0.021 \\
-0.034 \\
0.100
\end{array}\right\}+\left\{\begin{array}{c}
-17.023 \\
2.769 \\
-0.271
\end{array}\right\}
\end{array}
$$

Applying the similarity transformation (19) one obtains

$$
\left[\hat{K}^{\prime}\right]=\left[\begin{array}{ccc}
13.481 & 0 & 0 \\
0 & 46.470 & 0 \\
0 & 0 & 653.099
\end{array}\right]
$$


Table 2. Local attachment coordinates of the ligamentous structures according to Crowninshield et al. model.

\begin{tabular}{|c|c|c|c|c|c|c|c|c|}
\hline \multirow[b]{3}{*}{ Ligaments } & \multirow[b]{3}{*}{ Abbreviation } & \multicolumn{6}{|c|}{ Attachment sites } & \multirow{3}{*}{$\frac{\text { Material properties }}{K_{\mathrm{I}}\left(\frac{\mathrm{N}}{\mathrm{mm}}\right)}$} \\
\hline & & \multicolumn{3}{|l|}{ Femoral } & \multicolumn{3}{|l|}{ Tibial } & \\
\hline & & $X_{f}(\mathrm{~mm})$ & $Y_{f}(\mathrm{~mm})$ & $Z_{f}(\mathrm{~mm})$ & $X_{t}(\mathrm{~mm})$ & $Y_{t}(\mathrm{~mm})$ & $Z_{t}(\mathrm{~mm})$ & \\
\hline Lateral Collateral & LC & 35 & 10 & 25 & 45 & 25 & -35 & 10.0 \\
\hline Post. Med. Collateral & MCP & -35 & 8 & 30 & -20 & 8 & -50 & 5.0 \\
\hline Ant. Med. Collateral & MCA & -35 & -7 & 30 & -20 & -7 & -40 & 10.0 \\
\hline Med. Coll. Oblique & $\mathrm{MCO}$ & -35 & 3 & 30 & -35 & 30 & -10 & 5.0 \\
\hline Deep Med. Coll. & DMC & -35 & 0 & 27 & -35 & 0 & -10 & 5.0 \\
\hline Ant. Post. Cruciate & APC & -5 & -3 & 20 & -5 & 25 & -5 & 31.3 \\
\hline Post. Post. Cruc. & PPC & -5 & 17 & 20 & 5 & 25 & -5 & 19.3 \\
\hline Ant. Fib. Ant. Cruc. & AAC & 7 & 8 & 25 & -7 & -5 & 0 & 22.5 \\
\hline Post. Fib. Ant. Cruc. & PAC & 5 & 4 & 25 & 0 & -2 & 0 & 26.3 \\
\hline Lat. Post. Cap. & LPC & 25 & 25 & 30 & 25 & 25 & -30 & 12.0 \\
\hline Med. Post. Cap. & MPC & -25 & 25 & 30 & -25 & 25 & -30 & 12.0 \\
\hline Post. Cap. Oblique & PCO & 25 & 25 & 30 & -25 & 25 & -30 & 3.0 \\
\hline Post. Cap. Oblique & $\mathrm{PCO}$ & -25 & 25 & 30 & 25 & 25 & -30 & 3.0 \\
\hline
\end{tabular}

$$
+\varepsilon\left[\begin{array}{ccc}
-545.937 & 0 & 0 \\
0 & 716.399 & 0 \\
0 & 0 & -170.397
\end{array}\right]
$$

From the matrix $[\hat{E}]$, we can compute the dual Euler parameters. In particular, we obtain the following results

$$
\begin{aligned}
& \hat{e}_{0}=0.707+\varepsilon(0.069) \\
& \hat{e}_{1}=0.018+\varepsilon(-5.007) \\
& \hat{e}_{2}=0.005+\varepsilon(-1.377) \\
& \hat{e}_{3}=0.0003+\varepsilon(0.0698)
\end{aligned}
$$

while the dual angle $\hat{\theta}$ and the screw axes $\{\hat{h}\}$ can be written as follows

$$
\begin{aligned}
& \hat{\theta}=1.571+\varepsilon(-0.194) \\
& \{\hat{h}\}=\left\{\begin{array}{c}
0.0255+\varepsilon(-7.0776) \\
0.0072+\varepsilon(-1.9465) \\
0.0004+\varepsilon(0.0988)
\end{array}\right\}
\end{aligned}
$$

\section{Model of Crowninshield et al.}

The second model of knee joint considered in this work is the model proposed by Crowninshield et al. $^{2}$; also in this model, the tibia and the femur are considered as two rigid bodies, but now is the tibia the fixed element, while the femur is considered to be free. The stiffness of the knee joint is due essentially to the ligamentous structures. The ligaments are modeled as 13 spring elements. In particular, the ACL is made up of two elements, as the PCL. The LCL is represented by one fiber while the medial one is modeled by four elements. The posterior capsular structure is modeled by four elements. These elements are represented in Figure 5. The original paper does not report the ligament stiffness. For this reason, the stiffness adopted in our computations are the same of those reported in Ref. 1, as summarized in Table 2.

The coordinates of the tibial and femural insertion sites of the ligaments are determined by the examination of anatomical specimens and represent an average of values obtained from seven different subjects. These coordinate values are reported in Table 2.

The values of the stiffness coefficients of the spring elements used to model the different ligamentous structure were assumed according to the data available in literature.

Applying the algorithm just presented in the previous section, we are able to compute the stiffness dual matrix $[\hat{K}]$ which is defined as

$$
\begin{aligned}
{[\hat{K}]=} & {\left[\begin{array}{ccc}
51.201 & 27.766 & -8.950 \\
27.766 & 103.713 & -58.162 \\
-8.950 & -58.162 & 579.207
\end{array}\right] } \\
& +\varepsilon\left[\begin{array}{ccc}
-1407.359 & -2216.934 & 5579.926 \\
-2216.934 & 465.663 & 1878.596 \\
5579.926 & 1878.596 & 941.696
\end{array}\right]
\end{aligned}
$$

The computation of eigenvalues $\hat{\lambda}$ and eigenvectors $\{\hat{e}\}$ led us to the following results

$$
\begin{aligned}
& \hat{\lambda}_{1}=38.803+\varepsilon(-388.135) \\
& \hat{\lambda}_{2}=586.497+\varepsilon(-217.364) \\
& \hat{\lambda}_{3}=108.820+\varepsilon(555.499)
\end{aligned}
$$




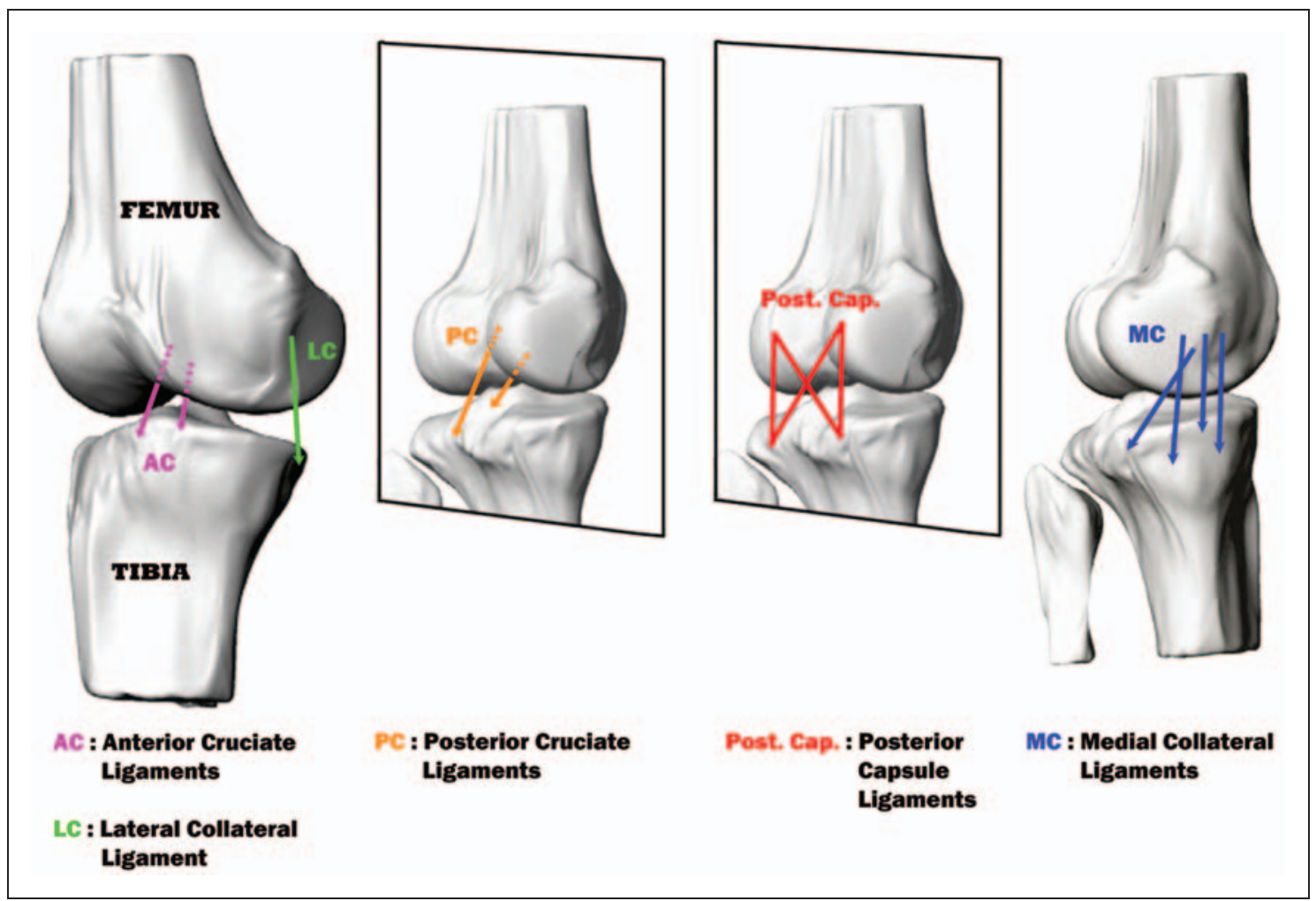

Figure 5. Schematic representation of the ligaments by line vectors - model of Crowninshield.

$$
\begin{aligned}
& \left\{\hat{e}_{1}\right\}=\left\{\begin{array}{c}
0.909 \\
-0.415 \\
-0.029
\end{array}\right\}+\left\{\begin{array}{c}
10.586 \\
23.536 \\
-5.2013
\end{array}\right\} \\
& \left\{\hat{e}_{2}\right\}=\left\{\begin{array}{c}
-0.0229 \\
-0.121 \\
0.992
\end{array}\right\}+\left\{\begin{array}{c}
11.132 \\
4.449 \\
0.798
\end{array}\right\} \\
& \left\{\hat{e}_{3}\right\}=\left\{\begin{array}{c}
0.416 \\
0.902 \\
0.119
\end{array}\right\}+\left\{\begin{array}{c}
-22.529 \\
11.443 \\
-7.928
\end{array}\right\}
\end{aligned}
$$

Applying the similarity transformation

$$
\left[\hat{K}^{\prime}\right]=[\hat{E}]^{T}[\hat{K}][\hat{E}]
$$

where $[\hat{E}]$ is the dual matrix whose columns are represented by the eigenvectors just computed, we obtain the diagonal form of the stiffness dual matrix as follows

$$
\begin{aligned}
{\left[\hat{K}^{\prime}\right]=} & {\left[\begin{array}{ccc}
38.803 & 0 & 0 \\
0 & 586.497 & 0 \\
0 & 0 & 108.820
\end{array}\right] } \\
& +\varepsilon\left[\begin{array}{ccc}
388.135 & 0 & 0 \\
0 & 217.364 & 0 \\
0 & 0 & -555.499
\end{array}\right]
\end{aligned}
$$

From the matrix $[\hat{R}]$, we can compute the dual Euler parameters; in particular, we obtain the following results

$$
\begin{aligned}
& \hat{e}_{0}=0.691+\varepsilon(1.286) \\
& \hat{e}_{1}=0.033+\varepsilon(-3.915) \\
& \hat{e}_{2}=0.161+\varepsilon(-6.573) \\
& \hat{e}_{3}=-0.142+\varepsilon(4.755)
\end{aligned}
$$

while the dual angle $\hat{\theta}$ and the screw axes $\{\hat{h}\}$ can be written as follows

$$
\begin{aligned}
& \hat{\theta}=1.617+\varepsilon(-3.557) \\
& \{\hat{h}\}=\left\{\begin{array}{l}
0.045+\varepsilon(-5.335) \\
0.223+\varepsilon(-8.710) \\
-0.197+\varepsilon(6.241)
\end{array}\right\}
\end{aligned}
$$

\section{Conclusions}

Given two bodies connected by means of linear springs, the proposed methodology allows the definition of a dual diagonal stiffness matrix. This approach provides a theoretical framework for comparing the numerical stiffness obtained by adopting different human knee models. In fact, the results are decoupled from the Cartesian coordinate system adopted for expressing the stiffness matrix. The usefulness of this framework is self evident when the stiffness matrix follows from different experimental 
measurement setup. In fact, one can compare different knee stiffness linear models in a framework independent from the Cartesian reference system.

Another element of novelty of this paper is the application of the algorithm for the similarity transform of a dual stiffness matrix. The application focused on two linearly elastic models known in literature. The models compared have ligaments with the same stiffness but different points of attachment. Hence, the elastic forces will have different lines of actions. This explains the difference of results. The order of magnitude of the estimated dual stiffness values is somewhat similar.

The validity of our results is limited to small displacements in the neighborhood of an equilibrium configuration. In fact, the experimental evidence demonstrates that, for large rotation angles, the stiffness is not linear anymore and more complex constitutive equations are required.

\section{Funding}

This research received no specific grant from any funding agency in the public, commercial, or not-for-profit sectors.

\section{References}

1. Abdel-Rahaman EM and Hefzy MS. Three-dimensional dynamic behaviour of the human knee joint under impact loading. Med Eng Phys 1998; 20: 276-290.

2. Crowninshield R, Pope MH and Johnson RJ. An analytical model of the knee. J Biomech 1976; 9: 397-405.

3. Abdel-Nasser AM, Brown MA and Shabana AA. Study of the ligament tension and cross-section deformation using nonlinear finite element/multibody system algorithms. Multibody Syst Dyn 2010; 23: 227-248.

4. Guess TM. Forward dynamics simulation using a natural knee with menisci in the multibody framework. Multibody Syst Dyn 2012; 28: 37-53.

5. McFaull SR and Lamontagne M. In vivo measurement of the passive viscoelastic properties of the human joint. Hum Mov Sci 1998; 17: 139-165.

6. Akbar M, Farhmand F, Jafari A, et al. A detailed and validated three dimensional dynamic model of the patellofemoral joint. ASME J Biomech Eng 2012; 134: 041005.

7. Saevarsson SK, Romeo CI and Anglin C. Are static and dynamic kinematics comparable after total knee arthroplasty? J Biomech 2013; 46: 1169-1175.
8. Ball RS. Theory of screws. Cambridge: Cambridge University Press, 1900.

9. Dimentberg FM. The screw calculus and its applications in mechanics. Technical report, AD 680993. Virginia: Clearinghouse for Federal and Scientific Technical Information, 1968.

10. Fasse ED. Some applications of the screw theory to lumped-parameter modeling of visco-elastically coupled rigid bodies. In: Proceedings of a symposium commemorating the legacy, works and life of Sir Robert Stawell Ball, Upon the 100th Anniversary of A Treatise on the Theory of Screws, 9-11 July 2000, University of Cambridge, Trinity College, UK.

11. Loncaric J. Geometrical analysis of compliant mechanisms in robotics. $\mathrm{PhD}$ thesis, Division of Applied Science, Harvard University, Cambridge, MA, June 1985.

12. Loncaric J. Normal form of stiffness and compliance matrices. IEEE J Robot Automat 1987; 3: 567-572.

13. Pennestrì E and Valentini PP. Linear dual algebra algorithms and their application to kinematics. In: Bottasso CL (ed) Multibody dynamics computational methods and applications. Springer Verlag, 2009, vol. 12, pp. 207-230.

14. Enea C, Pennestrì E, and Valentini PP. A model for computing the dual stiffness matrix of the human knee joint. In Proceedings of the 2nd joint international conference on multibody system dynamics, Stuttgart, Germany, 29 May-1 June 2012.

15. Cheng HH. Computer-aided displacement analysis of spatial mechanisms using the $\mathrm{Ch}$ programming language. Adv Eng Software 1995; 23: 163-172.

16. Cheng HH. $C$ for engineers and scientists: an interpretative approach. New York: McGraw-Hill, 2009.

17. Ciblak N and Lipkin H. Asymmetric Cartesian stiffness for the modeling of compliant robotic systems. Robot Kinemat Dyn Controls 1994; 72: 197-204.

18. Cheng HH. Scientific computing in the Ch programming language. Sci Program 1993; 2: 49-75.

19. Cheng HH. Handling of complex numbers in the Ch programming language. Sci Program 1993; 2: 76-106.

20. Pennestrì E and Stefanelli R. Linear algebra and numerical algorithms using dual numbers. Multibody Syst Dyn 2007; 18: 323-344.

21. Pennestrì E and Valentini PP. Dual quaternions as a tool for rigid body motion analysis: a tutorial with an application to biomechanics. Arch Mech Eng 2010; LVII(2): 187-205. 